CERN/PPE/95-195

August 1, 1995

\title{
Measurement of Momentum and Angular Distribution of Punchthrough Muons at the RD5 Experiment
}

\author{
RD5 Collaboration, CERN, Geneva, Switzerland
}

Aachen $^{1}$ - Amsterdam (NIKHEF-H) ${ }^{2}-$ Boston University ${ }^{3}-$ Budapest $^{4}-$

UC Los Angeles ${ }^{5}-$ UC Riverside ${ }^{6}-$ CERN $^{7}-$ Firenze $^{8}-$ Helsinki $^{9}-$ Helsinki(SEFT) ${ }^{10}-$

Louisiana State University ${ }^{11}$ - Madrid (CIEMAT) ${ }^{12}$ - Madrid (Universidad Autónoma) ${ }^{13}$ - Moscow ${ }^{14}$ - Nijmegen ${ }^{15}$ - Padova ${ }^{16}$ - Roma "La Sapienza"17 - Roma "Tor Vergata"18 -

Roma "Terza Universitá"19 - SUNY at Stony Brook ${ }^{20}$ - Turku (Åbo Akedemi) ${ }^{21}$ -

Vienna (HEPHY) $)^{22}$ - Warsaw University ${ }^{23}$ - Inst. for Nucl. Studies, Warsaw ${ }^{24}-$ Zürich $^{25}$

C. Albajar ${ }^{13}$, M. Andlinger ${ }^{22}$, A. Arefiev ${ }^{14}$, C. Bacci ${ }^{19}$, Gy. L. Bencze ${ }^{4}$, R. Bergman ${ }^{15}$,

A. Bizzeti ${ }^{8}$, C. Brouwer ${ }^{15}$, R. Cardarelli ${ }^{18}$, P. Casoli $^{16}$, S. Centro ${ }^{16}$, F. Ceradini ${ }^{19}$,

E. Choumilov ${ }^{14}$, D. Chrisman ${ }^{5}$, G. Ciapetti ${ }^{17}$, C. Civinini ${ }^{8}$, D. Cline ${ }^{5}$, R. D'Alessandro ${ }^{8}$,

M. Della Negra ${ }^{7}$, E. Denes ${ }^{4}$, A. Di Ciaccio ${ }^{18}$, W. Dominik ${ }^{23}$, H. Faissner ${ }^{1}$, A. Ferrando ${ }^{12}$, M.C. Fouz ${ }^{12}$, W. Gorn ${ }^{6}$, M. Górski ${ }^{24}$, A. Hervé ${ }^{7}$, A. Iglesias ${ }^{12, a}$, M. Juntunen ${ }^{10}$,

V. Karimäki ${ }^{10}$, R. Kinnunen ${ }^{9}$, A. Kluge ${ }^{22}$, Yu. Kolotaev ${ }^{14}$, M. Konecki ${ }^{23}$, A.C. König ${ }^{15}$,

J. Królikowski ${ }^{23}$, F. Lacava ${ }^{17}$, J.G. Layter ${ }^{6}$, P. Le Coultre ${ }^{25}$, C. Lyndon ${ }^{11}$, A. Malinin ${ }^{14}$,

G. Margutti ${ }^{17}$, R. Martinelli ${ }^{16}$, L. Martinez-Laso ${ }^{12}$, R. McNeil ${ }^{11}$, A. Meneguzzo ${ }^{16}$,

M. Meschini ${ }^{8}$, T. Moers ${ }^{1}$, M. Mohammadi-Baarmand ${ }^{20}$, A. Nisati ${ }^{17}$, D. Orestano ${ }^{17}$,

K. Österberg ${ }^{21}$, S. Otwinowski ${ }^{5}$, E. Petrolo ${ }^{17}$, M. Pimiä ${ }^{10}$, V. Pojidaev ${ }^{8}$, C. L. A. Pols ${ }^{15}$,

L. Pontecorvo ${ }^{17}$, P. Porth ${ }^{22}$, E. Radermacher ${ }^{7}$, B. Razen ${ }^{1}$, H. Reithler ${ }^{1}$, R. Ribeiro ${ }^{7}$,

A. Rojkov ${ }^{14}$, A. Sanjari ${ }^{20}$, R. Santonico ${ }^{18}$, P. Sartori ${ }^{16}$, H. Schwarthoff ${ }^{1}$, C. Seez ${ }^{7, b}$,

J. Shank ${ }^{3}$, B.C. Shen ${ }^{6}$, M. Szeptycka ${ }^{24}$, F. Szoncso ${ }^{22}$, H. Teykal ${ }^{1}$, H. Tolsma ${ }^{2}$,

H. Tuchscherer ${ }^{1, c}$, J. Tuominiemi ${ }^{9}$, T. Tuuva ${ }^{10}$, H. van der Graaf ${ }^{2}$, S. Veneziano ${ }^{17}$,

M. Verzocchi ${ }^{17}$, G. Vesztergombi ${ }^{4}$, H. Wagner ${ }^{1}$, G. Walzel $^{22}$, T. Wijnen ${ }^{15}$, G.W. Wilson ${ }^{6}$, G. Wrochna ${ }^{7}$, C.-E. Wulz ${ }^{22}$, L. Zanello ${ }^{17}$, and P. Zotto ${ }^{16, d}$.

\begin{abstract}
The momentum and angular distributions of punchthrough muons have been measured after a $10 \lambda$ calorimeter using an iron toroid magnet with $1.5 \mathrm{~T}$ as spectrometer. The calorimeter was inside a variable magnetic field of 0 to $3 \mathrm{~T}$. The incident momentum of the $\pi^{-}$beam ranged from 20 to $300 \mathrm{GeV} / c$. Measurements were also done at some beam momenta for $\pi^{+}, \mathrm{K}^{+}$and p. The results are compared with Monte Carlo predictions. A parameterization for the momentum spectrum of punchthrough muons was derived from the data.
\end{abstract}

To be submitted to Z. Phys. C

\footnotetext{
${ }^{a}$ Doctoral student from Univ. of Santiago de Compostela, Spain.

${ }^{b}$ Visitor from Imperial College, London, U.K.

${ }^{c}$ Now at University of Alabama, Tuscaloosa, USA.

${ }^{d}$ Now at Dipartimento di Física del Politecnico, Milano, Italy.
} 


\section{Introduction}

Designing muon detectors for the Large Hadron Collider (LHC) requires accurate knowledge of muon backgrounds. Muon chamber performance is influenced by the particle rate; track reconstruction is influenced by muon induced secondaries near the muon track and the muon trigger threshold is restricted by background particle rate and momentum distribution.

The muon detectors at LHC are located behind thick absorbers, which reduce the particle rate and make muons very attractive for trigger and event selection purposes. Almost all interesting phenomena, like production of Higgs particles, SUSY particles, etc., provide decay channels with one or more muons in the final state. These muons normally have a high transverse momentum, which distinguishes them from background. The background consists mainly of prompt muons from charm and bottom decays [1]. Other muon background sources are $\pi$ and K decays in the central tracking volume, uncorrelated neutrons or gammas and hadronic punchthrough.

The RD5 experiment at CERN [2] was designed to study several aspects of muon detection at a $\mathrm{LHC}$ detector. The influence of muon induced electromagnetic secondaries on the muon track measurement was studied with a wide variety of muon chamber prototypes, which were tested at RD5 [3]. The main physics goal of RD5 was the measurement of hadronic punchthrough. The results on total rate of punchthrough particles have been published recently [4].

While the momentum and angular distributions of prompt and decay muons can be readily calculated, for muons generated in a hadronic cascade it is more complicated and involves supplementary shower generators. In the RD5 experiment we measured these distributions for punchthrough muons reaching a depth of at least $20 \lambda$.

In this paper we describe the experimental setup in section 2 and the event selection and reconstruction algorithm for the momentum analysis in section 3 . The results are presented in section 4 as absolute momentum spectra and as spectra relative to the incident beam momentum. In section 5 we present the angular distributions of punchthrough muons. The results are compared with Monte Carlo predictions.

\section{The RD5 experiment}

The RD5 experiment is located at the H2 beam of the CERN SPS North Area. The experimental setup is described in detail in $[2,4]$ and the references therein. Here, we give only a short description of the components essential for the momentum analysis. The RD5 detector was designed to simulate a segment of the CMS detector [5], having a tracker, a calorimeter and muon measurement stations. The setup includes two magnets, a superconducting magnet (M1) with a maximum field of $3 \mathrm{~T}$, and an iron toroidal magnet (M2) operated at $1.5 \mathrm{~T}$. The magnet $\mathrm{M} 1(B \mathrm{~d} l=5.2 \mathrm{Tm})$, which contains a tracking calorimeter (TRACAL), simulates the strong solenoidal field of CMS $(B \mathrm{~d} l \approx 12 \mathrm{Tm})$, while the magnet M2 simulates the CMS return yoke and is used as a muon spectrometer. The M1 and M2 fields are horizontal (z-axis) and perpendicular to the beam. The field components in $x$ and y-direction are below $1 \%$ in the region near the beam line. The field orientation of the first half of M2 is reversed to the field orientation in M1, in the second half the orientations are the same. 
The beam particle first traverses two multiwire proportional chambers (MWPC), which measure the beam position and are used to reject multi-particle events.

The calorimeter, TRACAL, consists of stainless steel plates $\left(0.8 \times 1.0 \mathrm{~m}^{2}\right)$ interleaved with $0.6 \times 0.8 \mathrm{~m}^{2}$ Honeycomb Strip Chambers (HSC) $[6]$ in $22 \mathrm{~mm}$ gaps. The first 12 steel plates are $40 \mathrm{~mm}$ thick, the last 12 plates are $80 \mathrm{~mm}$ thick, thus totaling to about 9 nuclear interaction lengths $\lambda$ and 25 HSC layers. A lead brick of $1 \lambda$ thickness $\left(21 \mathrm{X}_{0}\right)$ is located in front of the calorimeter to represent the absorption of an electromagnetic calorimeter. The HSCs were read out with cathode strips in the bending plane and with drift wires in the non-bending plane. For this analysis we used only the hit wire multiplicity in the non-bending plane to reject incoming muons from beam contamination.

Three muon measurement stations are located in front of, inside and behind the absorber magnet. The muon stations consist of drift chambers [7] and Resistive Plate Chambers (RPC) [8]. The muon drift chambers (taken from the UA1 experiment) are used for precise measurement of particle trajectories in the RD5 spectrometer. The positions of the chambers were surveyed and the alignment was checked with high momentum muons without magnetic field. The spatial and angular errors of the track segments in each chamber are about $1 \mathrm{~mm}$ and $1 \mathrm{mr}$, respectively, in each projection. The intrinsic track resolution of a single chamber, without the alignment error, is about $250 \mu \mathrm{m}$ and was used for the track reconstruction at station level. Muon Station 1, being just behind TRACAL and M1 is at a depth of $10 \lambda$. Muon Stations 2 and 3 are at depths of $20 \lambda$ and $32 \lambda$, respectively.

The momentum resolution of the muon spectrometer is $17 \%(19 \%)$ for $20 \mathrm{GeV} / c$ muons, $22 \%(26 \%)$ for $100 \mathrm{GeV} / c$ muons and deteriorates to $33 \%(54 \%)$ for $300 \mathrm{GeV} / c$ muons. The numbers in brackets give the resolution if only two of the three muon stations are used for the momentum fit.

The RD5 trigger system consists of two scintillators for the beam time definition (overlapping area $\left.10 \times 10 \mathrm{~cm}^{2}\right)$ and additional scintillators for beam size definition $\left(2 \times 2 \mathrm{~cm}^{2}\right.$ or $\left.4 \times 4 \mathrm{~cm}^{2}\right)$. Another system of scintillators, together with a $4 \times 4 \mathrm{~m}^{2}$ large RPC wall, form a veto system against beam halo particles. It is ensured that there are no other particles crossing the veto wall within $\pm 2 \mu$ s around the triggering particle. This veto system also covered the beam line, thus rejecting double particle events in the $2 \mu$ s time window. However, the efficiency of the RPCs in the veto wall was about $95 \%$, thus not all halo particles were rejected.

Two exclusive trigger conditions were used for the punchthrough measurements: a minimum bias trigger (MB), consisting of the beam definition triggers with an anticoincidence of the veto counter, and a $10 \lambda$ trigger, consisting of the MB trigger in coincidence with a $2 \times 2 \mathrm{~m}^{2} \mathrm{RPC}$ at the first muon station.

A Čerenkov counter, being a standard $\mathrm{H} 2$ beam equipment, was used when running with positive pions, kaons and protons in order to distinguish various kinds of particles.

\section{The Data Sample and the Analysis Method}

The data used for the momentum analysis were taken during the 1993 runs. The analysis included data sets with different energies and particle types, taken with the MB trigger and the $10 \lambda$ trigger. The reduction of the $10 \lambda$ trigger was calculated by counting the number of events in the MB data, which also satisfied the $10 \lambda$ trigger condition. This reduction 


\begin{tabular}{|c|c|c|c|c|c|c|c|c|}
\hline \multirow{2}{*}{$\begin{array}{c}\text { momentum } \\
{[\mathrm{GeV} / c]}\end{array}$} & \multicolumn{8}{|c|}{ particle type } \\
\hline & \multicolumn{2}{|l|}{$\pi^{-}$} & \multicolumn{2}{|l|}{$\pi^{+}$} & \multicolumn{2}{|c|}{$\mathrm{K}^{+}$} & \multicolumn{2}{|c|}{ proton } \\
\hline & \multicolumn{8}{|c|}{ event statistics, M1 field $0 \mathrm{~T}$} \\
\hline 20 & $2,031,000$ & 294 & & & & & & \\
\hline 30 & $1,224,000$ & 355 & $1,307,000$ & 394 & & & & \\
\hline 50 & $1,618,000$ & 1033 & & & & & & \\
\hline 100 & 687,000 & 1121 & 365,000 & 522 & & & 862,000 & 1096 \\
\hline 200 & 255,000 & 880 & & & & & & \\
\hline \multirow[t]{2}{*}{300} & 325,000 & 1502 & 217,000 & 869 & & & 208,000 & 873 \\
\hline & \multicolumn{8}{|c|}{ 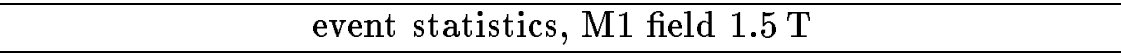 } \\
\hline 30 & 866,000 & 230 & & & & & & \\
\hline 100 & 735,000 & 1047 & & & & & & \\
\hline \multirow[t]{2}{*}{300} & 208,000 & 857 & & & & & & \\
\hline & \multicolumn{8}{|c|}{ event statistics, M1 field $3 \mathrm{~T}$} \\
\hline 20 & $2,254,000$ & 175 & & & & & & \\
\hline 30 & 941,000 & 152 & $2,022,000$ & 201 & & & & \\
\hline 50 & $1,055,000$ & 276 & & & 667,000 & 311 & & \\
\hline 75 & & & & & & & 880,000 & 324 \\
\hline 100 & 791,000 & 708 & 165,000 & 106 & 195,000 & 217 & 302,000 & 171 \\
\hline 200 & 277,000 & 530 & & & & & & \\
\hline 300 & 322,000 & 812 & 114,000 & 261 & & & 141,000 & 329 \\
\hline
\end{tabular}

Table 1: Event statistics for momentum analysis. The first column of each particle type shows the number of min. bias events corresponding to the analysed data set (see text). The second column of each particle type shows the number of signal events with at least one track that passes all cuts.

factor ranged from 20 for a $30 \mathrm{GeV} / c$ pion beam down to 2 for a $300 \mathrm{GeV} / c$ pion beam. In Table 1 we present the event statistics used in the momentum analysis. The number of events listed are the sum of MB and $10 \lambda$ trigger data, after the number of $10 \lambda$ triggers has been normalized by multiplying with the reduction factor. This sum represents the number of MB events one would have to take in order to get the same event statistics reaching a depth of $10 \lambda$. For each of these data sets we took also some data with a muon beam for background estimation and detector calibration.

The $\pi^{-}$beam contained up to $8 \%$ muons at the low beam energies, thus causing a major problem for the analysis. The electron contamination was found to be negligible. The positive beam consisted of a mixture of different particles, which had to be selected by the Cerenkov counter. However, muons and pions could not be separated and therefore the $\pi^{+}$beam contained a similar muon contamination as the $\pi^{-}$beam. The Cerenkov counter, located $130 \mathrm{~m}$ upstream, could also not completely reject the muons from kaon decays. Thus, the only pure hadron beam was the proton beam.

To reject the muon contamination, we defined a variable called activity, which is the wire multiplicity in TRACAL folded with the lateral spread of the hit wires (see [4] for 
details). In Fig. 1 the activity distribution of $30 \mathrm{GeV} / c$ and $300 \mathrm{GeV} / c$ pions and muons is shown as an example; the vertical scale is arbitrary. The cut used to separate pions from muons ranged from $A=7.5$ for $30 \mathrm{GeV} / c$ to $A=40$ for $300 \mathrm{GeV} / c$. The separating power of this variable is poor for low beam energies, but increases with the momentum. For each beam energy the signal losses due to this cut were calculated by subtracting the normalized activity distribution of the muon beam from the activity distribution of the pion beam and counting the number of pion events that fail the cut. The muon distribution was normalized to have the same number of entries, with activity less than 1.5 , as the pion distribution. The signal loss was estimated to be $14.6 \pm 0.5 \%$ for $30 \mathrm{GeV} / c$ beam and $2.4 \pm 0.1 \%$ for $300 \mathrm{GeV} / c$ beam. This signal loss was confirmed by scanning a fraction of the rejected events for several energies. The signal loss is almost cancelled by the remaining background from beam halo (see 3.1) and was not corrected for.

Using this algorithm, a hadron, penetrating the whole calorimeter without interaction or decaying before the first interaction, is identified as "muon" and rejected. Our detector did not provide any means to separate these events from the muon background. This topic is discussed in more detail in sec. 4.1.

An additional muon background arose from hadrons which began to shower upstream of the calorimeter. At low energies the beam had a wide profile and hadrons showered frequently in the shielding and trigger devices just upstream of the detector $(10 \%$ of the selected events for a $30 \mathrm{GeV} / c$ beam). These secondaries had a high chance to decay, giving this additional muon background. These events were characterized by having a large number of tracks in one or both MWPC chambers. Events having two or more tracks in both MWPCs, or having more than two tracks in any of the MWPCs, were rejected as multiparticle events. The MWPC cut removed almost all of these events. On the other hand, due to noise and e.m. secondaries, the MWPC gave wrong multiplicities from time to time. This effect was measured with muon data, and the MWPC efficiency was found to be $\left(91.8 \pm 0.2_{\text {stat }} \pm 1_{\text {sys }}\right) \%$, independent of beam energy and also stable with time. Since this cut was only applied for selected events, the MWPC efficiency had to be corrected for.

\subsection{Momentum Fit}

To fit the momentum of the punchthrough muons, we selected only events with at least one track in the second muon station, thus requiring the particle to penetrate at least $20 \lambda$ of absorber. One has to keep this fact in mind when looking at our results, where we give the distributions for a reference depth of 0 or $10 \lambda$. For each track in the second muon station we searched for matching tracks in the first and third muon station, requiring to find at least one. These track candidates were then fitted using the GEANE package of the GEANT V3.21 library [9, 10].

The fitted track was rejected as beam halo, if it did not point back to the calorimeter. It was checked, that this cut caused no signal loss, however, halo muons penetrating the calorimeter were not rejected. These tracks are not distinguishable from beam contamination background and were accounted for by scanning (see next paragraph).

The final data samples were scanned (100 events of each sample). The fraction of background events identified in the scanned sample is about $12.5 \%$ for $30 \mathrm{GeV} / c \pi^{-}$beam and drops to about $2 \%$ for $300 \mathrm{GeV} / c \pi^{-}$beam. These are nearly the same numbers as for the signal loss due to the activity cut. When comparing the momentum spectra of the 


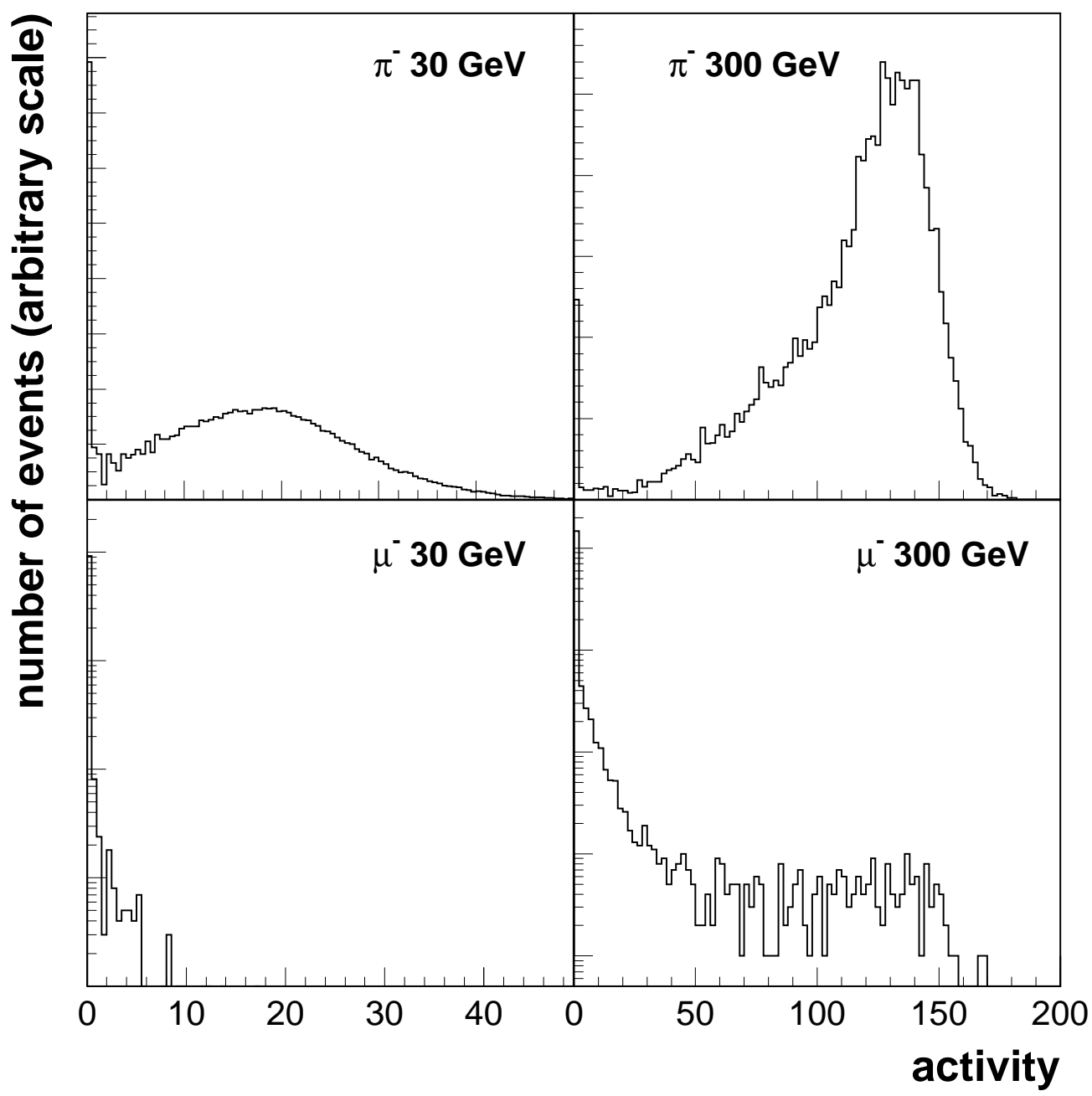

Figure 1: Example of activity distribution for $30 \mathrm{GeV} / c$ and $300 \mathrm{GeV} / c$ pions and muons. The vertical scale is arbitrary. The cut values used to separate pions from muons was 7.5 for $30 \mathrm{GeV} / c$ and 40 for $300 \mathrm{GeV} / c$. The muon distributions are shown with a logarithmic scale. Note also the different scales on the ordinate for the two beam energies. 
lost signal events and the identified background events, one finds that both have the same shape and are nearly flat over the whole momentum range. (For this comparison we used the relative momentum and combined the data of all beam momenta). Thus the background subtraction and signal loss correction almost cancel each other. We therefore made no background subtraction and signal loss correction, but added an adequate systematic error (from $5 \%$ for $30 \mathrm{GeV} / c$ down to $1 \%$ for $300 \mathrm{GeV} / c$ ).

After the fit and applying the cuts we produced the spectra shown in the next section. If there was more than one track reconstructed, we only took the one with the best $\chi^{2}$-value per degree of freedom. This was done because most of the multi-track events stem from reconstruction ambiguities, and only a few are real multi-track events, which was checked by scanning.

\subsection{Geometrical Acceptance}

The final distributions had to be corrected for the geometrical acceptance of the RD5 spectrometer. The absorber magnet covers an area of $80 \mathrm{~cm}$ above and below the beam. Tracks that are bent outside of this fiducial region were rejected at Station 2 and 3. At Station 1 , all tracks outside of the calorimeter boundaries were rejected. To study this geometrical acceptance, we enlarged the calorimeter and the absorber magnet in the simulation to cover the whole area of the muon chambers. The geometrical loss was found to be well below $10 \%$ for M1 off. However, this acceptance affects only low momentum particles (below $10 \mathrm{GeV} / \mathrm{c}$ ) and changes the distributions shown here only slightly. We did not correct for this effect.

This is not true if the M1 magnet is operated at $3 \mathrm{~T}$. In that case the acceptance is only about $50 \%$ of all particles. Again, particles with momenta above $10 \mathrm{GeV} / c$ are not affected. To be able to compare the distributions with the magnetic field at 0 and $3 \mathrm{~T}$ we corrected the momentum distributions that were taken with the field on for the geometrical acceptance. The correction factors were calculated with simulated muons traversing the field. The factors were 3.55 for momenta below $5 \mathrm{GeV} / c, 1.68$ for $7 \mathrm{GeV} / c$ and 1.0 for $10 \mathrm{GeV} / c$. We used a linear interpolation of these values for the correction.

\subsection{Simulation}

For comparison with our measurements we produced simulated data with GEANT, using GHEISHA [11] as hadronic shower generator. To generate a reasonable number of muons reaching $20 \lambda$ we had to tune the program for speed. Only in the last $8 \mathrm{~cm}$ of TRACAL all particles were tracked in the calorimeter, to get approximately the correct distribution of tracks in the first muon station. In the rest of TRACAL only particles with momentum above $3 \mathrm{GeV} / c$ were tracked. Since the momentum threshold for muons penetrating the first half of the absorber magnet is $3 \mathrm{GeV} / c$, we did not lose signal events by this modification.

The simulation speed was further increased by switching off most of the electromagnetic processes (like Compton scattering and pair production). Since almost all muons are generated by hadronic processes, this modification does not change the signal. However, the number of electrons is largely reduced, so the distribution of background tracks is not correct. This only slightly affects the momentum analysis. We used this very fast Monte Carlo to increase the statistics for $\pi^{-}$beam, with the M1 magnet off, for $100 \mathrm{GeV} / c$ and $300 \mathrm{GeV} / c$ beam momentum. Two third of the total statistics of these two data sets were 
generated using this modification. The simulation of the $50 \mathrm{GeV} / c$ beam was completely performed using the very fast Monte Carlo.

The simulated data were treated in the same way as the real data, except for the activity cut and the MWPC cut, which were not applied. Table 2 gives an overview of the available Monte Carlo data.

\begin{tabular}{|c|c|c|c|c|c|c|c|c|}
\hline \multirow{2}{*}{$\begin{array}{c}\text { momentum } \\
{[\mathrm{GeV} / c]}\end{array}$} & \multicolumn{8}{|c|}{ particle type } \\
\hline & \multicolumn{2}{|l|}{$\pi^{-}$} & \multicolumn{2}{|l|}{$\pi^{+}$} & \multicolumn{2}{|c|}{$\mathrm{K}^{+}$} & \multicolumn{2}{|c|}{ proton } \\
\hline & \multicolumn{8}{|c|}{ event statistics, M1 field $0 \mathrm{~T}$} \\
\hline 30 & 250,000 & 93 & \multirow{4}{*}{60,000} & \multirow{4}{*}{74} & & & & \\
\hline 50 & 150,000 & 123 & & & & & & \\
\hline 100 & 180,000 & 193 & & & & & & \\
\hline \multirow[t]{2}{*}{300} & 60,000 & 201 & & & & & & \\
\hline & \multicolumn{8}{|c|}{ event statistics, M1 field $3 \mathrm{~T}$} \\
\hline 30 & 250,000 & 64 & & & & & & \\
\hline 100 & 60,000 & 59 & 60,000 & 83 & 60,000 & 92 & 60,000 & 30 \\
\hline 300 & 20,000 & 48 & & & & & & \\
\hline
\end{tabular}

Table 2: Event statistics for simulation. The first column of each particle type shows the number of generated events. The second column shows the number of events with at least one track passing all cuts.

\section{Momentum of Punchthrough Muons}

The momentum spectra of punchthrough muons for various beam energies and particle types are shown in Figures 3 to 7 . We corrected these spectra for the inefficiencies of the detector and the fit. These include:

- Trigger efficiency of the $10 \lambda$ trigger: $99.0 \% \pm 0.3 \%$.

- Track segment reconstruction efficiency in one muon station (position and direction dependent); $79-91 \%$, typical error: $2 \%$.

- Matching efficiency, of track segments between two muon stations, and fit efficiency, momentum dependent: $90-50 \%$, typical error: $2 \%$.

- MWPC efficiency (i.e. percentage of identified single track events): $91.8 \% \pm 1.2 \%$ (see above).

In Fig. 2 we show the product of all these efficiencies as function of the muon momentum. The errors were treated as systematic errors, their sum is also shown. These efficiencies were calculated using muon data with momenta of 10 to $300 \mathrm{GeV} / c$, simulated muons and data generated using the fast Monte Carlo.

In Figs. 3 and 4 we present the momentum spectra of punchthrough muons for different beam energies of $\pi^{-}$and $\pi^{+}$beams with the M1 field at 0 and $3 \mathrm{~T}$. The probabilities, 


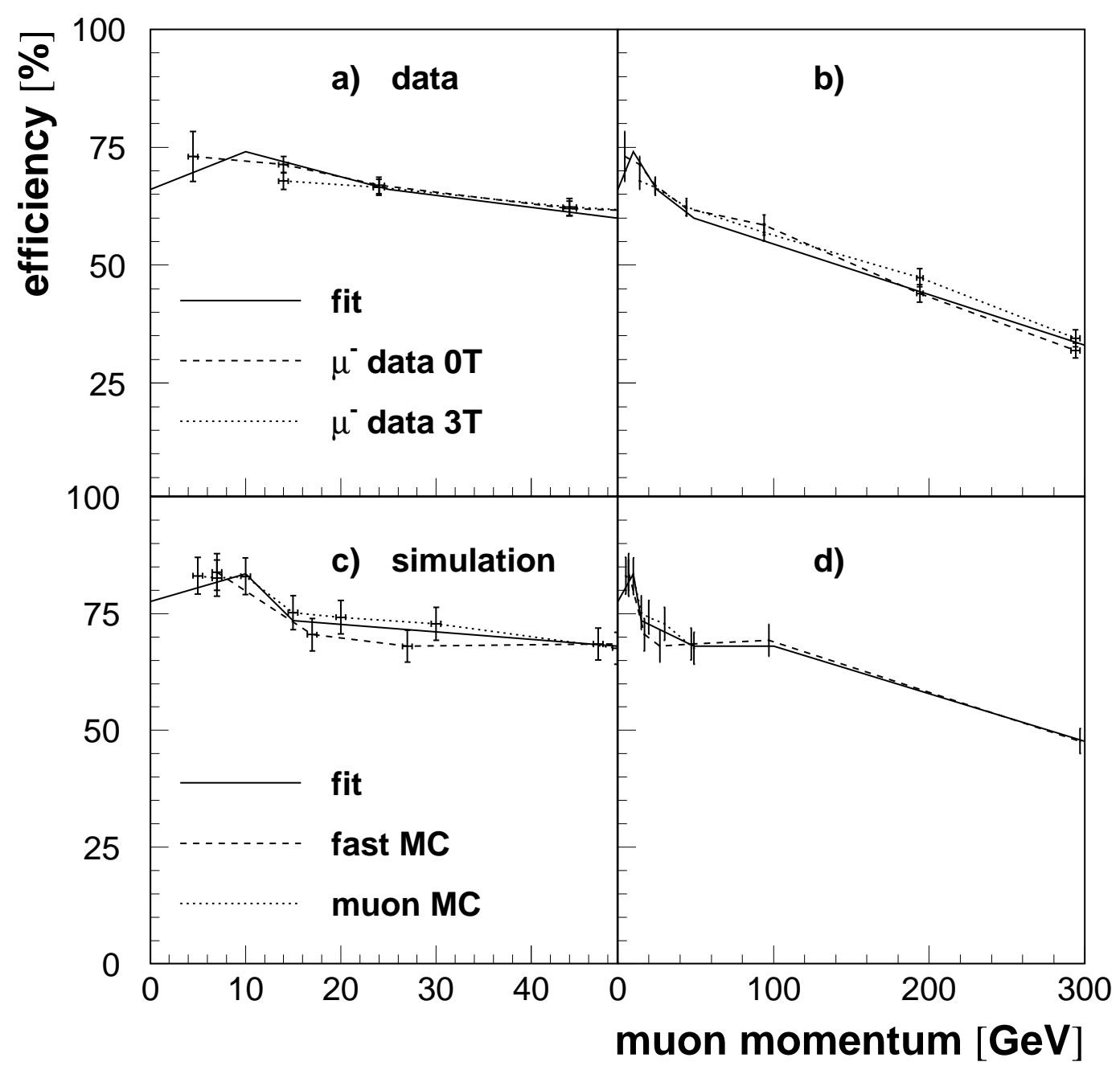

Figure 2: The total efficiency for the momentum fit as function of the muon momentum at a depth of $10 \lambda$. Figs. a) and b) show the efficiency for real data, Figs. c) and d) show the efficiency for simulated data. Figs. a) and c) show an enlarged view of the low momenta, while Figs. b) and d) cover the whole momentum range. The solid curves were used for the correction of the momentum spectra. 
integrated over the muon momentum, are consistent with the total punchthrough probabilities measured earlier [4]. In the figures, negative and positive momenta correspond to negatively and positively charged punchthrough muons, respectively. The hole in the center of the distribution is caused by the $3 \mathrm{GeV} / c$ momentum threshold for muons penetrating the absorber magnet from the momentum reference plane at $10 \lambda$ to the second muon station at a depth of $20 \lambda$. The upper momentum threshold is, of course, given by the beam momentum. Momentum values above the beam momentum are caused by the momentum resolution of the detector.

The charge asymmetry observed in each spectrum is explained by the fact that the leading particle in a hadronic shower carries the charge of the incident hadron. Subsequent decay of this particle will lead to a secondary muon with the same charge as the incident hadron. A naive calculation of the average charged multiplicty of the hadron shower after the first interaction, with the constraint that the net charge of the shower has to be the same as the charge of the incident particle, gives indeed the charge asymmetries observed.

The errors of the geometrical acceptance correction, described in the previous section, are not included in the plots. Even without these errors the distributions for field on and field off are equal within the errors. We did not expect an effect of the field, except for the additional geometrical loss due to the bending, since the bending power of the field is too weak to trap particles of $3 \mathrm{GeV} / c$ and above.

In Fig. 5 we compare the fast Monte Carlo with the data. For this plot we combined the data with field off and field on for each momentum, after applying the acceptance correction to the data with field on. The distributions are compatible within errors. The errors of the simulated distributions are quite large, due to the limited statistics.

For beam energies of 30 and $50 \mathrm{GeV}$ we observe an enhancement of simulated $\mu^{-}$with momenta just below the beam momentum. We explain this by primary pion decay. As stated earlier, the activity cut removes decay muons from our data, even if the hadron decays inside the calorimeter before the first interaction. The simulated data, however, contain these events, since the cut is not applied there. More evidence for this fact is given in the next section.

In Fig. 6 we compare the momentum spectra taken with the same beam momentum $(100 \mathrm{GeV} / c)$, but with different beam particles. To show that the distributions of $\pi^{-}$and $\pi^{+}$beam data are nearly identical, if the different charge is accounted for, we changed the sign of the charge for the $\pi^{-}$data. For better comparison, we did not plot the error bars. The distributions are nearly identical within the errors, except for the proton beam data. This distribution has significantly less muons with momenta above $30 \mathrm{GeV} / c$. This effect is expected, since there are no first generation decays possible. On the other hand, we would expect the spectrum of the kaon beam data to be slightly harder than the pion beam data. This may not be visible in the plots due to the lack of statistics. The comparison between different beam particle types at other beam energies also did not show any significant difference, except for the protons.

\subsection{Parameterization of Muon Momentum}

In Fig. 7 we show the secondary momentum relative to the beam momentum for four different beam momenta of $\pi^{-}$beams. The simulation is also included. A fit of a double exponential function was applied to all four data sets, excluding the first bins where thresh- 


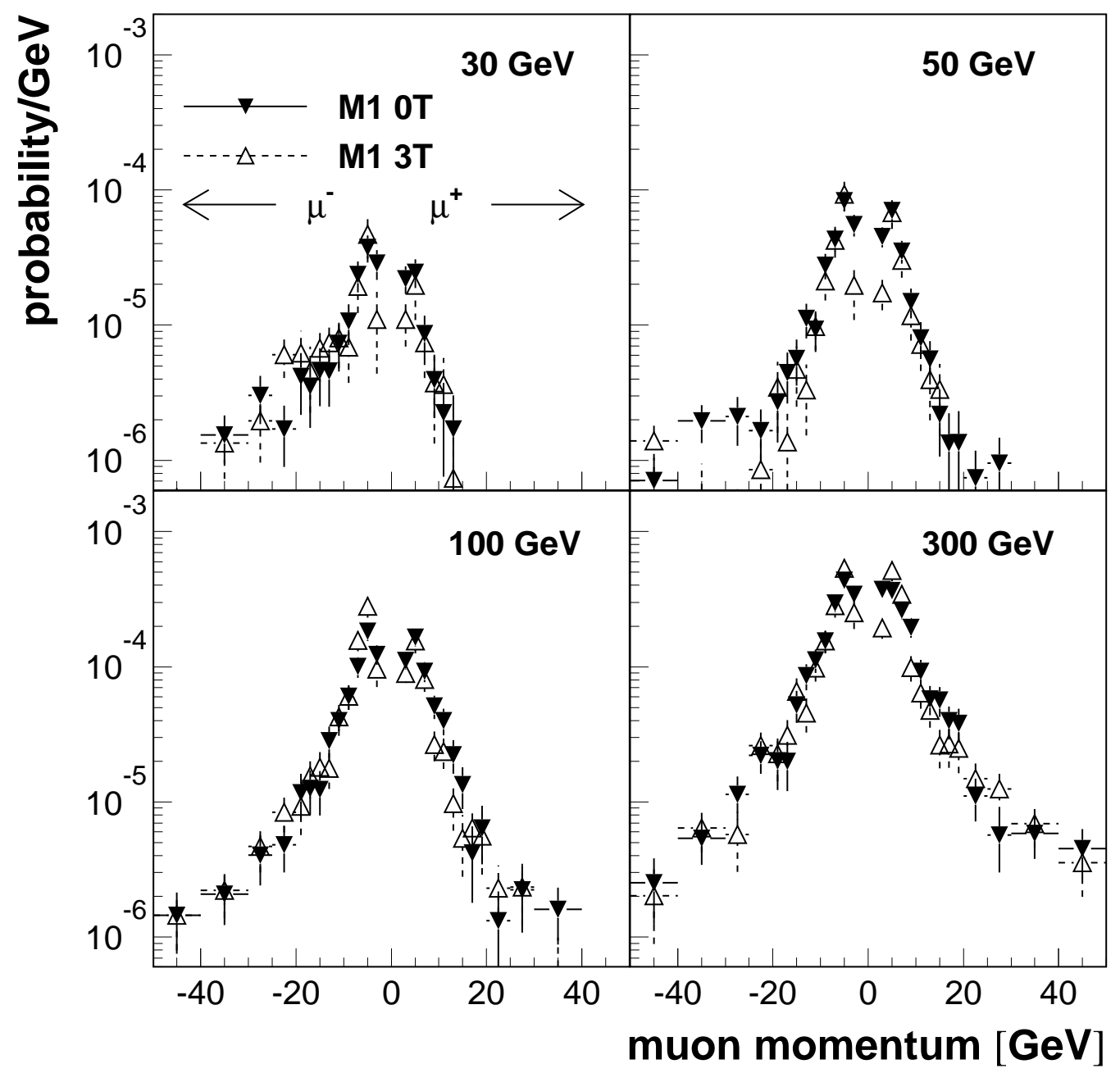

Figure 3: Measured momentum of punchthrough muons from $\pi^{-}$beams with four different energies, at a depth of $10 \lambda$. Data with the M1 magnet off (solid triangle) and at full field (open triangle) are shown. The negative muons are plotted with negative momentum. 


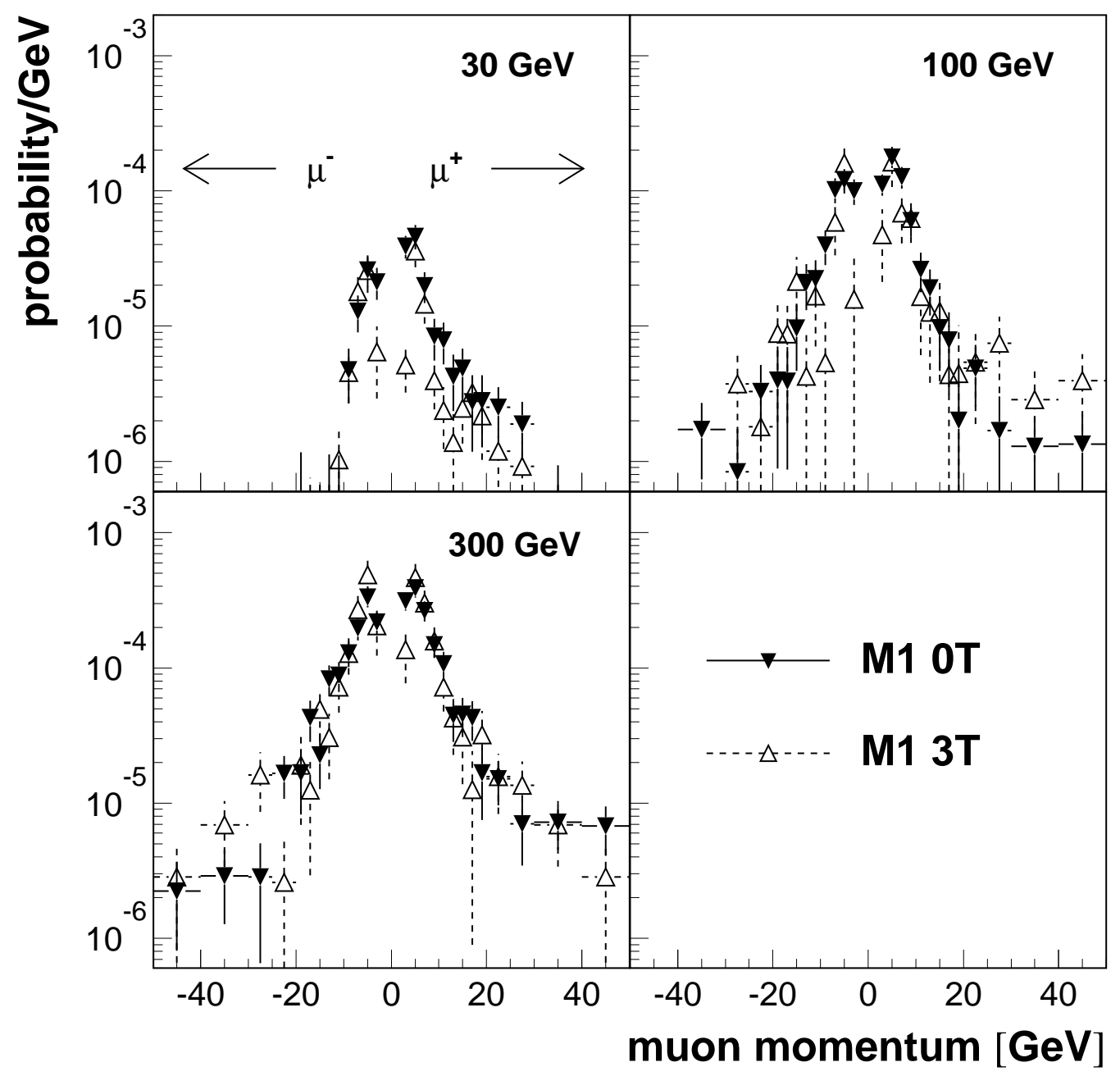

Figure 4: Measured momentum of punchthrough muons from $\pi^{+}$beams with three different energies, at a depth of $10 \lambda$. Data with the M1 magnet off (solid triangle) and at full field (open triangle) are shown. The negative muons are plotted with negative momentum. 


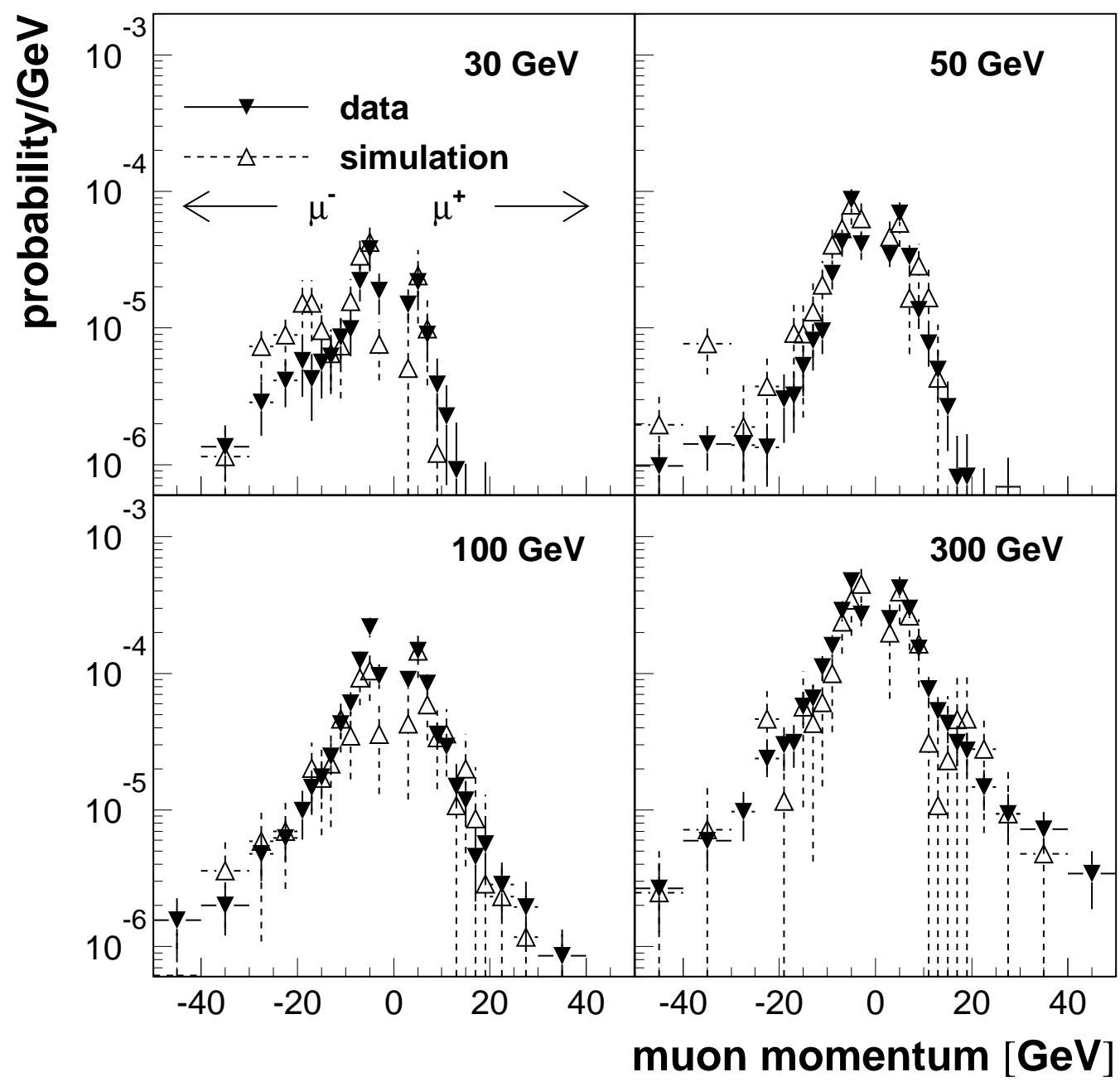

Figure 5: Comparison of $\pi^{-}$data with the GEANT simulation. The data with field off and field on were combined. 


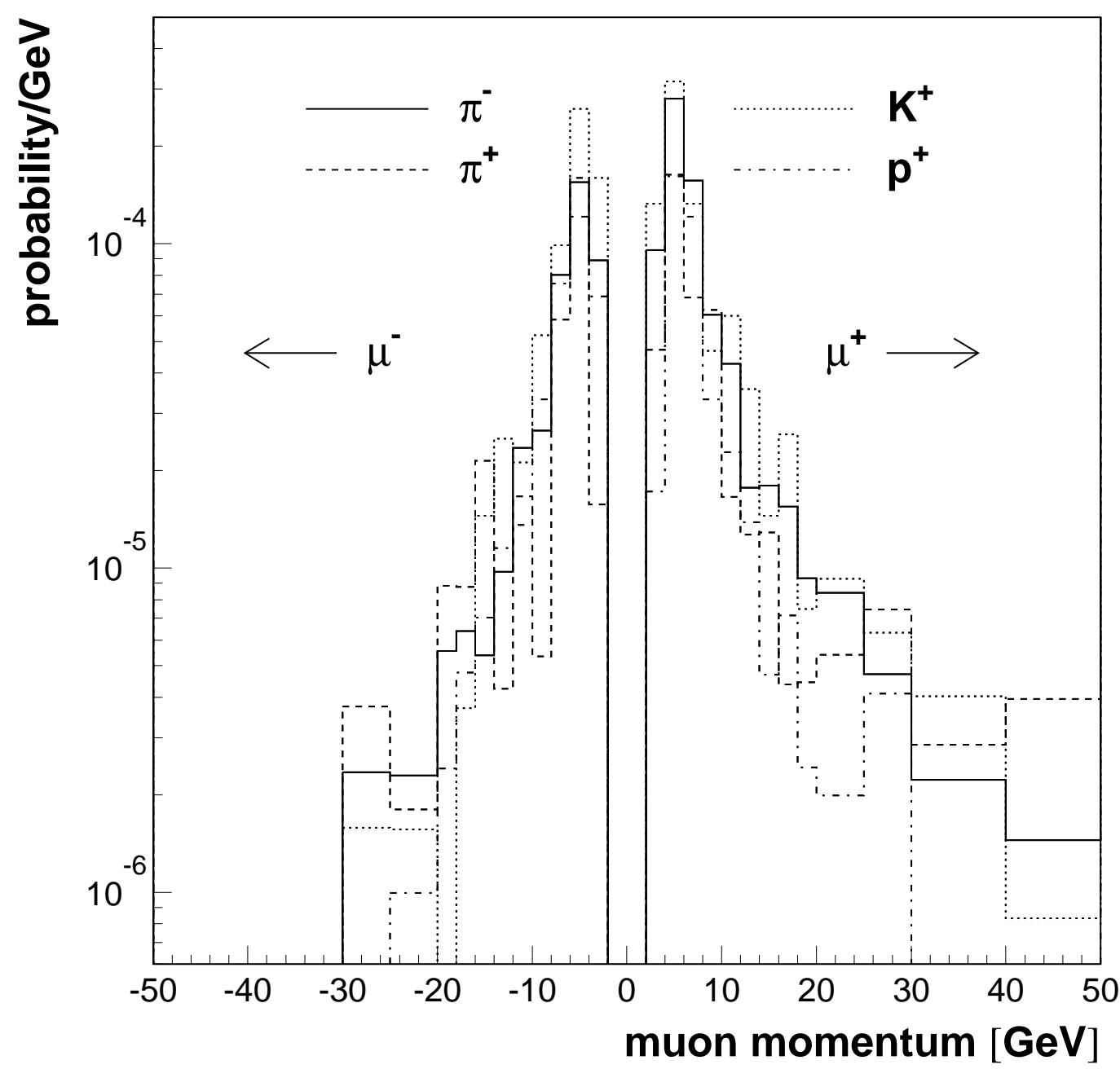

Figure 6: Comparison of punchthrough muon momentum from four different beams. All data shown here were taken with $100 \mathrm{GeV} / c$ beam momentum and with the M1 magnet at $3 \mathrm{~T}$. For better comparison the sign of the charge was changed for the $\pi^{-}$beam data and the error bars are not included. 
old effects are visible. As can be seen in the figure, both the data and the simulation are well described by this function, except for the primary pion decay, as already mentionned in the previous section.

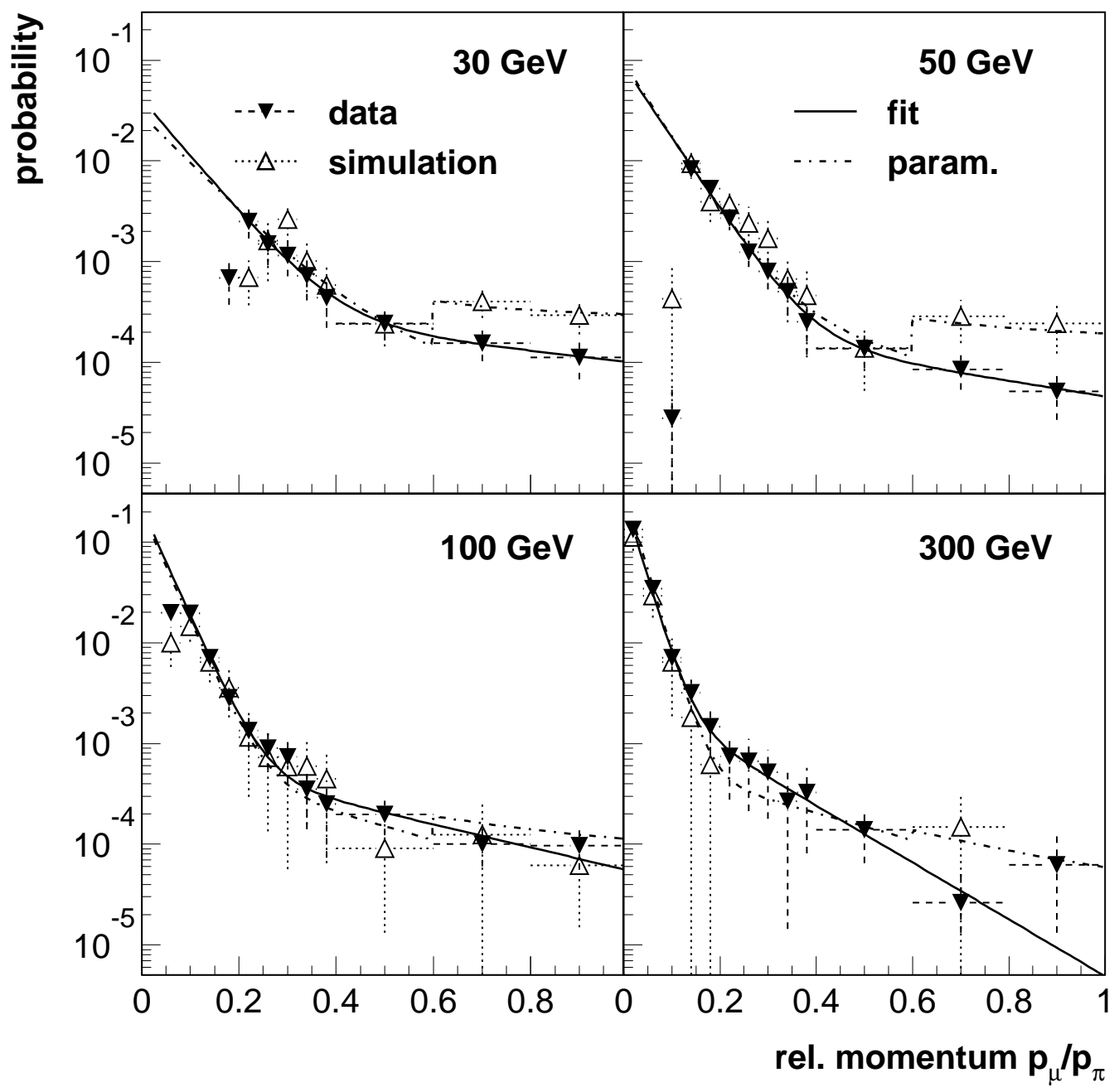

Figure 7: Secondary muon momentum at a depth of $0 \lambda$, both charges combined, relative to the incident beam momentum for four $\pi^{-}$beam energies. The solid triangles are the data, the solid lines respresent a fit of a double exponential to the data. The hollow triangles are the simulation. The dash-dotted line is the overall parameterization including primary pion decay (see text).

From these four fits we derived an overall parameterization $f$, valid for all beam momenta above $30 \mathrm{GeV} / c$ :

$$
f=f_{1}+f_{2}+g ; \quad f_{i}=a_{i} \exp \left(b_{i} z\right) .
$$

Where $z$ is the relative momentum. The function $g$ describes the primary pion decay and was simply calculated from the decay probability in the first interaction length: $\frac{\lambda}{c \tau_{\pi} \gamma}=$ 
$2.3 \cdot 10^{-2} \frac{m_{\pi}}{E_{\pi}}$; the momenta of the decay muons are equally distributed in the range from roughly two third of the beam momentum to the full beam momentum.

$$
g= \begin{cases}0 & z<0.6 \\ 2.5 \times 2.3 \cdot 10^{-2} \frac{m_{\pi}}{E_{\pi}} & z \geq 0.6\end{cases}
$$

The factor 2.5 is needed to normalize the integral of $g$ over $z$ to the correct decay probability.

The parameters $a_{2}$ and $b_{2}$ are the average values of the four fits, the parameters $a_{1}$ and $b_{1}$ are dependent on the beam momentum and can be parameterized as

$$
\begin{aligned}
& a_{1}(p)=\exp \left(\frac{k_{1}}{1+\log (p / 30 \mathrm{GeV})}\right) ; \\
& b_{1}(p)=k_{2}(1+\log (p / 30 \mathrm{GeV})) .
\end{aligned}
$$

The values are

$$
\begin{aligned}
& k_{1}=-3.57 \pm 0.67 \\
& k_{2}=-11.3 \pm 1.14 \\
& a_{2}=\exp (-7.27 \pm 1.39) \\
& b_{2}=-3.07 \pm 1.90 .
\end{aligned}
$$

The resulting function $f$ is also plotted in Fig. 7. It reproduces the pion data quite well. We also see a good agreement between our parameterization and the kaon data, if the kaon decay is handeled properly by replacing the function $g$ with $g_{\mathrm{K}}=4.9 \cdot 10^{-2} m_{\mathrm{K}} / E_{\mathrm{K}}$. The muon spectra from proton beams are also well described for the low muon momenta $(z<0.3)$, for higher momenta the parametrization is above the measured spectra.

The parameterization can be used instead of a full simulation by simply replacing incoming hadrons with muons using this function. The gain in simulation speed is a factor of 360 when compared with the fast Monte Carlo, which was already tuned for speed. We expect the parameterization to be about 7000 times as fast as a full shower simulation. However, it should be noted, that this parameterization was derived from data taken at an absorber depth of $20 \lambda$. At a depth of $10 \lambda$, for example, we observe a discrepancy between a full simulation and the parameterization for muons below the $3 \mathrm{GeV} / c$ threshold. The parameterization returns correct distributions at shallower depths only if a proper momentum cut is used.

In Fig. 8 we present the 30 and $100 \mathrm{GeV} / c \pi^{-}$beam data for negative muons ( $\mathrm{a}$ and $\mathrm{b}$ ) and positive muons ( $c$ and $d$ ) separately. The parameterization plotted here is

$$
\begin{aligned}
& f_{-}=0.5 f_{1}+f_{2}+g ; \\
& f_{+}=0.5 f_{1} .
\end{aligned}
$$

For positive beams the charge index has to be reversed. It can be seen, that the data is well described by the parameterization.

\section{Angular Distribution of Punchthrough Muons}

The projected angle of the tracks in the bending plane (i.e. the $x-y$-plane of the RD5 coordinate system) is shown in Figs. 9 and 10 for three different beam momenta. The first figure shows the data taken with the magnet M1 off and the second with the M1 field at $3 \mathrm{~T}$. The angle and momentum is given for a depth of $10 \lambda$. The angle in the transverse 


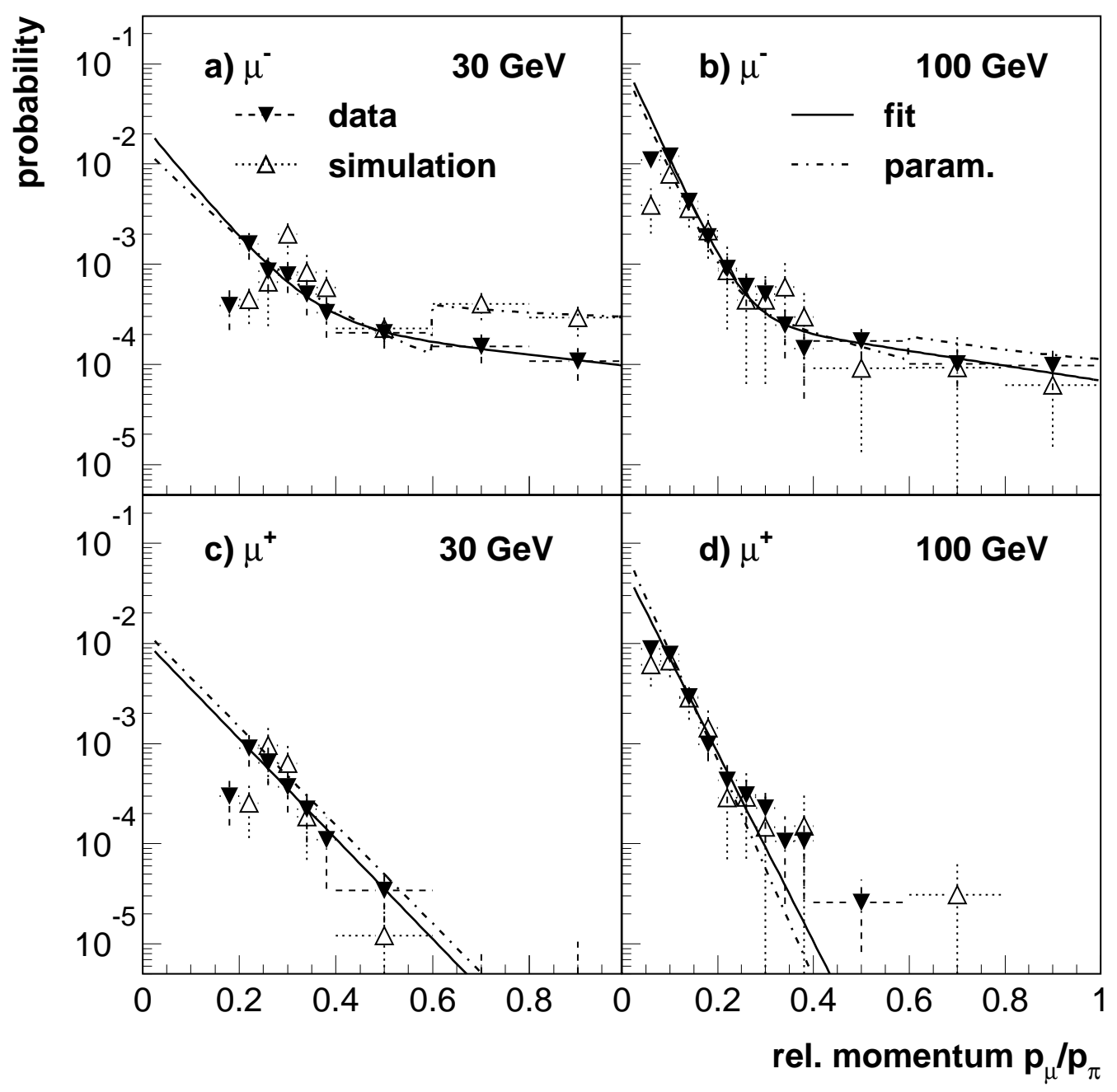

Figure 8: Secondary muon momentum relative to the incident beam momentum for two $\pi^{-}$ beam energies at a depth of $0 \lambda$, both muon charges separated. 
plane was also measured (not shown). It is independent of the field, as expected. The data has been corrected as described above. The errors shown include the systematic errors from these corrections, except for the geometrical acceptance correction, which affects only angles larger than 250 mrad.

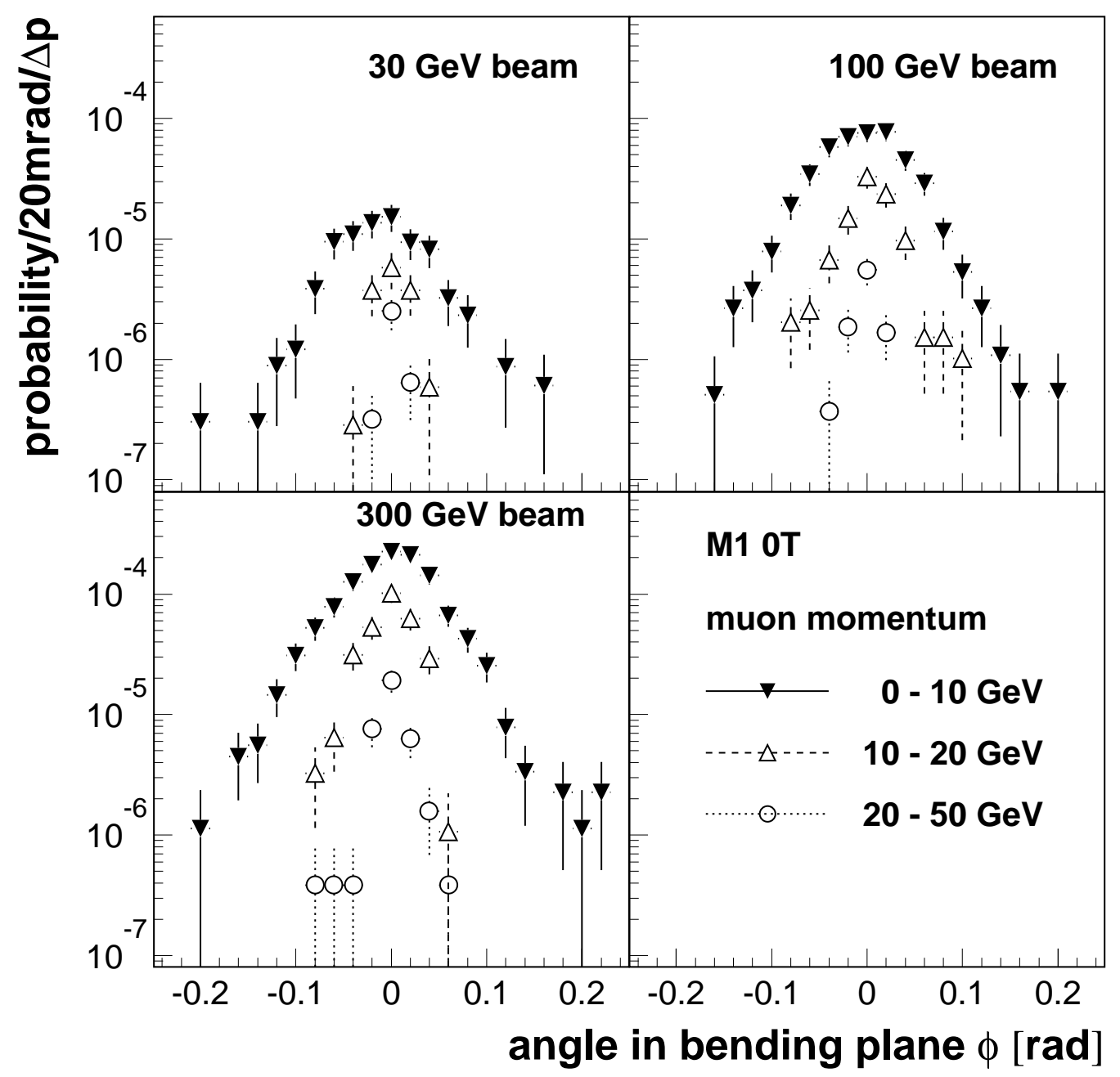

Figure 9: Angular distribution of secondary muons at a depth of $10 \lambda$ for three different beam momenta with the M1 field at $0 \mathrm{~T}$. The angle shown here is the projected angle in the bending plane. The distributions are shown for three intervals of the muon momentum.

In Fig. 9 the width of the distributions is determined by the hadronic processes in the calorimeter. It is narrower for higher momenta, as expected, however all distributions are slightly wider than they would be if multiple scattering would be the only process affecting the angular distribution. For comparison, the width of the angular distribution for multiple scattering is about $30(40) \mathrm{mrad}$ for $10 \mathrm{GeV} / \mathrm{c}$ muons and about $15(20) \mathrm{mrad}$ for $20 \mathrm{GeV} / c$ muons. The numbers in brackets are the widths of the corresponding distributions in Fig. 9.

In Fig. 10 the effect of the magnetic field is clearly seen. The asymmetry between negative and positive secondaries (negative particles have negative bending angles) is also visible. 


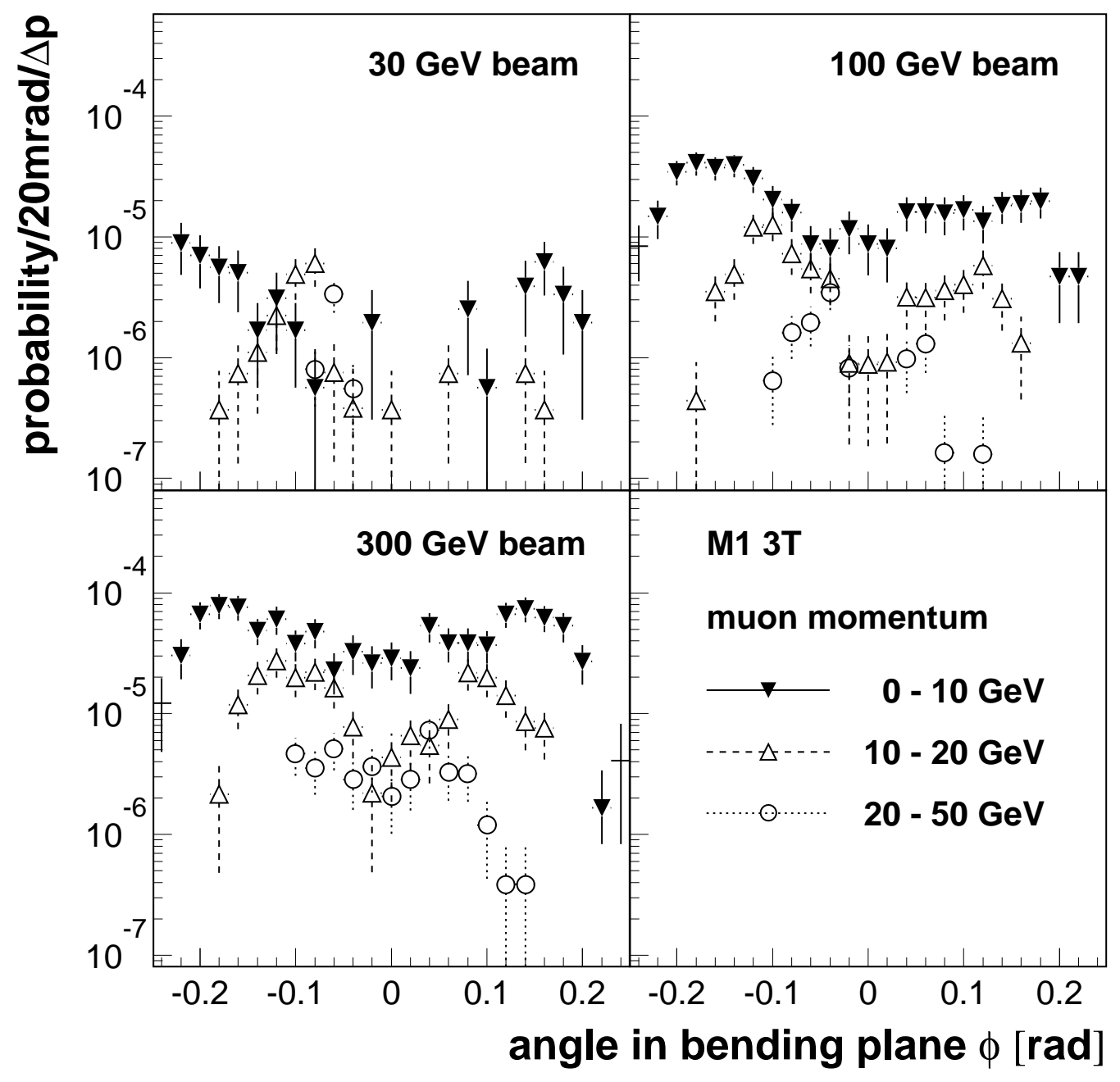

Figure 10: Angular distribution of secondary muons at a depth of $10 \lambda$ for three different beam momenta with the M1 field at $3 \mathrm{~T}$. The angle shown here is the projected angle in the bending plane. Positive muons have positive bending angles. The distributions are shown for three intervals of the muon momentum. 
The distribution for higher secondary momenta $(10-20 \mathrm{GeV} / c$ and $20-50 \mathrm{GeV} / c)$ is more asymmetric and also depends strongly on the incident beam momentum; this suggests that the high momentum secondaries stem from first generation decays, while the low momentum particles stem from second and third generation decays. For a beam momentum of $300 \mathrm{GeV} / \mathrm{c}$ the muons below $50 \mathrm{GeV} / c$ stem from second and higher generation decays; therefore the angular distribution is almost symmetric for a $300 \mathrm{GeV} / c$ beam. This was already seen in Fig. 8, where the asymmetry between the muon charges is only visible in the distribution of the high momentum muons, and their relative amount becomes less with higher beam momenta.

The distribution of $0-10 \mathrm{GeV} / c$ muons is affected by the geometrical acceptance, which gives a cutoff at $\pm 250 \mathrm{mrad}$. The outer boundaries of the other two distributions for each beam energy are given by the lower momentum threshold of their momentum intervals. The bending angles for $10 \mathrm{GeV} / c$ and $20 \mathrm{GeV} / c$ muons are $170 \mathrm{mrad}$ and $75 \mathrm{mrad}$, respectively.

\section{Conclusion}

We studied the momentum and angular spectra of punchthrough muons for different incident beam momenta and particles. The distributions for pion, kaon and proton beams with momenta of $20 \mathrm{GeV} / c$ to $300 \mathrm{GeV} / c$ agree with the Monte Carlo predictions of the GEANT/GHEISHA simulation program. Even when neglecting most of the electromagnetic processes in the simulation the generated muon spectrum is compatible with the experimental data.

If we correct for the geometrical acceptance, we see no effect of the magnetic field on the momentum distribution, due to the high momentum threshold of the muon spectrometer. However, the spatial and angular distribution of punchthrough muons is influenced by the field, as expected.

The relative momentum spectra of the secondary muons can be described by the sum of two exponential functions. One of these functions is logarithmically dependent on the beam momentum, the other is independent of it. This parameterization can be used in simulation studies to generate punchthrough muons behind thick absorbers.

\section{Acknowledgments}

For their support in the preparation and during the actual runs we thank S. Lazic, D. Peach, P. Petiot, J. Pothier, B. Powell and G. Waurick.

The authors also acknowledge the support of the following funding agencies:

- Deutsches Bundesministerium für Forschung und Technologie,

- Hungarian Academy of Science under grant OTKA-T-16823,

- Spanish CICYT under grants AEN92-0829 and AEN-93-0954-E,

- Instituto Nazionale di Fisica Nucleare, Italy,

- Texas National Research Laboratory Commission - award numbers RGFY92-118 and RGFY93-312A, 
- US Department of Energy - contract numbers DE-AC02-76CH00016, DE-FG05-91ER40617 and DE-FG02-92ER40697,

- Polish Committee for Scientific Research under grants KBN-PB-2 04229101 and KBN-SPUB-206/93.

\section{References}

[1] A. Nisati, Proc. LHC Workshop Aachen, 1990, eds. G. Jarlskog and D. Rein, CERN 90-10.

[2] M. Della Negra et al., CERN/DRDC/90-36, DRDC/P7. The RD5 Collaboration, CERN/DRDC 93-49.

[3] C. Albajar et al., Nucl. Instr. and Meth. A 364 (1995) 473.

[4] M. Aalste et al., Z. Phys. C60, (1993) 1-10. M. Aalste et al., Measurement and Simulation of Hadronic Shower Punchthrough in Magnetic Field, CERN/PPE 95-61, to be published in Z. Phys. C.

[5] CMS collaboration, Technical Proposal, CERN/LHCC 94-38, 1994.

[6] H. Van der Graaf, Nucl. Instr. and Meth. A 307 (1991) 220.

F. Bakker et al., Nucl. Instr. and Meth. A 330 (1993) 44.

[7] K. Eggert et al., Nucl. Instr. and Meth. 176 (1980) 217.

C. Albajar et al., Z. Phys. C44 (1989) 15.

[8] R. Santonico, R. Cardarelli, Nucl. Instr. and Meth. A 187 (1981) 377.

R. Cardarelli et al., Nucl. Instr. and Meth. A 263 (1988) 20.

[9] V. Innocente, M. Maire and E. Nagy, GEANE: Average Tracking and Error Propagation Package, July 1991, CERN Program Library.

[10] R. Brun, M. Hansroul and L.C. Lassalle, CERN DD/EE/82, 1982.

CERN Program Library Long Writeup W5013, CERN, 1993.

[11] H. Fesefeldt, Proc. 3rd Pisa Meeting on Advanced Detectors, Castiglione della Pescaia, 1986.

H. Fesefeldt, Nucl. Instr. and Meth. A 263 (1988) 114.

H. Fesefeldt et al., Nucl. Instr. and Meth. A 292 (1990) 279. 